H I G H L I G H T S

MOUSE GENETICS

\section{Keeping an ear out for modifiers}

Although the ability of some mouse genetic backgrounds to modify mutant phenotypes is well known, only a handful of the responsible modifier genes have been found. Now Ikeda et al. report a new mouse modifier gene - Mtapla - that rescues the hearing defects of C56BL/6J (B6) tubby (tub) mutant mice. Importantly, their findings strongly indicate that Mtapla interacts with proteins that establish and maintain neuronal synapses, and that in the B6 mouse strain, this association is disrupted by Mtap 1 a alleles that carry polymorphisms in functionally important regions of the gene.

The tub modifier (moth1) locus was mapped to chromosome 2 in 1999, after it was discovered that $t u b$ mice retain their hearing on some genetic backgrounds, such as on the AKR/J (AKR), CAST/Ei and 129P2/OlaHsd (129P2) mouse strains, while still developing other tub-associated phenotypes. So, to identify the responsible gene, Ikeda et al. finely mapped the moth 1 locus to an interval of $0.17 \mathrm{cM}$. By screening AKR-derived brain and eye cDNA libraries, Ikeda et al. then identified and mapped four additional genes to this region. One of these was Mtapla, which encodes the neuron-specific, microtubuleassociated protein 1a. On sequencing these cDNAs from the AKR and B6 strains, only Mtap 1a contained strain-specific sequence variations - 12 single-nucleotide changes that either altered amino acids or changed the length of an Ala-Pro repeat. The sequencing of CAST/Ei and 129P2 Mtap1a alleles also revealed ten amino-acid-changing polymorphisms between these strains and B6. To prove that Mtapla is indeed the modifier at mothl, the authors next introduced a 129P2-derived Mtapla transgene

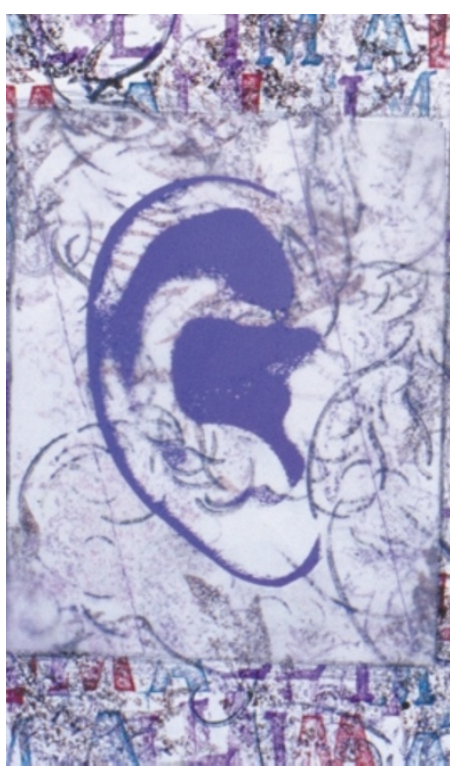

into B6 tub/tub mice, and almost completely rescued their hearing defects.

So, what is the function of Mtapla and how does it interact with tubby to protect against hearing loss? It appears that, in B6 mice, Mtapla function is compromised by polymorphisms in a region of the protein that resembles a guanylate kinase (GUK) binding site. GUK domains are present in a membrane-associated family of proteins that are crucial for the establishment of post-synaptic cytoarchitecture. Ikeda et al. found that one member of this family, Psd95, immunoprecipitates with Mtap1a in cerebellar extracts. Moreover, more Psd95 was present in complex with Mtap1a in AKRderived extracts than in $\mathrm{B} 6$ extracts, indicating that sequence polymorphisms in the B6 Mtap1a alleles might affect binding between these two proteins. Future studies should establish the physiological significance of this altered binding in neuronal synaptic function and hearing, and should reveal the still unexplained interaction between Mtapla and tubby itself.

Jane Alfred

\section{(2) References and links} ORIGINAL RESEARCH PAPER Ikeda, A. et al. Microtubule-associated protein $1 \mathrm{~A}$ is a modifier of tubby hearing (moth1). Nature Genet. 4 February (2002). DOI: 10.1038/ng838

FURTHER READING Nadeau, J. Modifier genes in mice and humans. Nature Rev. Genet. 2 165-174 (2001)

\section{IN BRIEF}

\section{QUANTITATIVE GENETICS}

Positional candidate cloning of a QTL in dairy cattle: identification of a missense mutation in the bovine DGAT1 gene with major effect on milk yield and composition.

Grisart, B. et al. Genome Res. 12, 222-231 (2002)

Most economically important traits in livestock are quantitative - they are influenced by many genes and by their interaction with the environment. This paper reports the positional cloning of a major quantitative trait locus (QTL) for milk yield and composition in dairy cattle. The authors believe that the magnitude of this QTL could be attributable to a single mutation in one gene: they have identified a non-conservative missense mutation in DGAT1, a strong positional candidate gene that lies in the mapped interval and which prevents lactation when it is knocked out in mice.

\section{SIGNAL TRANSDUCTION}

An allelic series at the PDGR $\alpha$ locus indicates unequal contributions of distinct signaling pathways during development.

Klinghoffer, R. A. et al. Dev. Cell 2, 103-113 (2002)

It is an aim of the signal-transduction field to determine which signalling proteins enable receptor tyrosine kinases (RTKs) to stimulate specific signalling pathways during development. To investigate this, Klinghoffer et al. generated knock-in mice that carry mutations in the Pdgfra (platelet-derived growth-factorreceptor- $\alpha$ ) gene, which selectively prevent this RTK from activating two of its target signalling proteins, PI3 kinase and Src kinase. Their findings show that loss of PI3 kinase activation leads to neonatal lethality due to impaired signalling in many cell types, whereas loss of Src activation affects only oligodendrocyte development.

\section{EVOLUTION}

Genetic variation for phenotypically invariant traits detected in teosinte: implications for the evolution of novel forms.

Lauter, N. \& Doebley, J. Genetics 160, 333-342 (2002)

Single-gene mutations can radically alter the morphology of an organism, but is this the mechanism by which taxa evolve, or do new character traits arise, as some propose, by many, small-effect genes that bring the population towards a certain threshold? The authors have tested a prediction of the latter idea by examining the standing genetic variation in traits that are invariant in teosinte, but in which teosinte differs from its modern relative - maize. The presence of phenotypically silent genetic variation in teosinte supports the multiple-gene theory that selection has acted on many loci during the domestication of this crop.

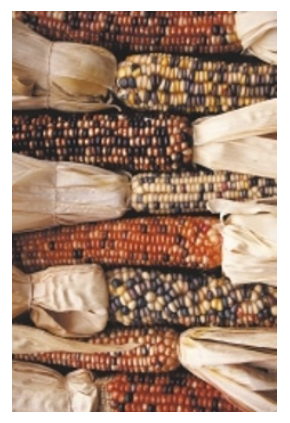

